\title{
Healthy lifestyle and the risk of pancreatic cancer in the EPIC study
}

Sabine Naudin ${ }^{1}$, Vivian Viallon ${ }^{1}$, Dana Hashim ${ }^{2}$, Heinz Freisling ${ }^{1}$, Mazda Jenab ${ }^{3}$, Elisabete Weiderpass $^{4,5,6,7}$, Flavie Perrier ${ }^{1}$, Fiona McKenzie ${ }^{8}$, H. Bas Bueno-de-Mesquita ${ }^{9,10,11}$, Anja Olsen $^{12}$, Anne Tjønneland ${ }^{12,13}$, Christina C. Dahm ${ }^{14}$, Kim Overvad ${ }^{14,15}$, Francesca Romana Mancini $^{16,17}$, Vinciane Rebours ${ }^{18,19}$, Marie-Christine Boutron-Ruault ${ }^{16,17}$, Verena Katzke ${ }^{20}$, Rudolf Kaaks ${ }^{20}$, Manuela Bergmann ${ }^{21}$, Heiner Boeing ${ }^{21}$, Eleni Peppa ${ }^{22}$, Anna Karakatsani $^{22,23}$, Antonia Trichopoulou ${ }^{22,24}$, Valeria Maria Pala ${ }^{25}$, Giovana Masala ${ }^{26}$, Salvatore Panico ${ }^{27}$, Rosario Tumino ${ }^{28}$, Carlotta Sacerdote ${ }^{29}$, Anne M. May ${ }^{30}$, Carla H. van Gils $^{30}$, Charlotta Rylander ${ }^{31}$, Kristin Benjaminsen Borch ${ }^{31}$, María Dolores Chirlaque López ${ }^{32,33}$, Maria-Jose Sánchez ${ }^{33,34}$, Eva Ardanaz ${ }^{33,35,36}$, J. Ramón Quirós ${ }^{37}$, Pilar Amiano 1 Exezarreta $^{33,38}$, Malin Sund ${ }^{39}$, Isabel Drake ${ }^{40}$, Sara Regnér ${ }^{40}$, Ruth C. Travis ${ }^{41}$, Nick 2 Wareham $^{42}$, Dagfinn Aune ${ }^{11,43,44}$, Elio Riboli ${ }^{11}$, Marc J. Gunter ${ }^{3}$, Eric J. Duell ${ }^{45}$, Paul 3 Brennan $^{46}$, Pietro Ferrari ${ }^{1^{*}}$

${ }^{1}$ Nutritional Methodology and Biostatistics Group, International Agency for Research on 6 Cancer, Lyon, France; ${ }^{2}$ Department of Hematology and Oncology, Tisch Cancer Institute, 7 Icahn School of Medicine at Mount Sinai, New York, NY; ${ }^{3}$ Nutritional Epidemiology Group, 8 International Agency for Research on Cancer, Lyon, France; ${ }^{4}$ Department of Community 9 Medicine, Faculty of Health Sciences, University of Tromsø, The Arctic University of Norway, 0 Tromsø, Norway; ${ }^{5}$ Department of Research, Cancer Registry of Norway, Institute of 1 Population-Based Cancer Research, Oslo, Norway; ${ }^{6}$ Department of Medical Epidemiology 2 and Biostatistics, Karolinska Institutet, Stockholm, Sweden; ${ }^{7}$ Genetic Epidemiology Group, 3 Folkhälsan Research Center and Faculty of Medicine, University of Helsinki, Helsinki, 4 Finland; ${ }^{8}$ Environment and Radiation section, International Agency for Research on Cancer, 25 Lyon, France; ${ }^{9}$ National Institute of Public Health and the Environment (RIVM), Bilthoven, 6 The Netherlands; ${ }^{10}$ Department of Gastroenterology and Hepathology, University Medical 7 Center, Utrecht, The Netherlands; ${ }^{11}$ Department of Epidemiology and Biostatistics, School of 8 Public Health, Imperial College London, United Kingdom; ${ }^{12}$ Danish Cancer Society Research 29 Center, Copenhagen, Denmark; ${ }^{13}$ Department of Public Health, Faculty of Health and Medical 30 Sciences, University of Copenhagen; ${ }^{14}$ Section for Epidemiology, Department of Public 1 Health, Aarhus University, Aarhus, Denmark; ${ }^{15}$ Department of Cardiology, Aalborg 2 University Hospital, Aalborg, Denmark; ${ }^{16}$ CESP, Fac. de médecine - Univ. Paris-Sud, Fac. de 33 médecine - UVSQ, INSERM, Université Paris-Saclay, 94805, Villejuif, France; ${ }^{17}$ Gustave 
Roussy, F-94805, Villejuif, FranceEPIC France; 18 Pancreatology Department, Beaujon

Hospital, DHU Unity, AP-HP, Clichy, and Paris-Diderot University, Paris, France; ${ }^{19}$ Inserm UMR1149, DHU Unity, and Paris-Diderot University, Paris, France; ${ }^{20}$ Division of Cancer Epidemiology, German Cancer Research Center (DKFZ), Heidelberg, Germany; ${ }^{21}$ German Institute of Human Nutrition Potsdam-Rehbrücke; ${ }^{22}$ Hellenic Health Foundation, Athens, Greece; ${ }^{23}$ Pulmonary Medicine Department, School of Medicine, National and Kapodistrian University of Athens, "ATTIKON" University Hospital, Haidari, Greece; ${ }^{24}$ School of Medicine, National and Kapodistrian University of Athens, Greece; ${ }^{25}$ Epidemiology and Prevention Unit, Fondazione IRCCS Istituto Nazionale dei Tumori, Milano, Italy; ${ }^{26}$ Cancer

Risk Factors and Life-Style Epidemiology Unit, Institute for Cancer Research, Prevention and

Clinical Network - ISPRO, Florence, ITALY; ${ }^{27}$ Department of Clinical and Experimental

Medecine, University Federico II, Naples, Italy; ${ }^{28}$ Cancer Registry and Histopathology

Department, Civic M.P.Arezzo Hospital, Ragusa, Italy; ${ }^{29}$ Unit of Cancer Epidemiology, Città della Salute e della Scienza University, Hospital and Center for Cancer Prevention (CPO), Turin, Italy; ${ }^{30}$ Julius Center for Health Sciences and Primary Care, University Medical Center Utrecht, Utrecht University, Utrecht, The Netherlands; ${ }^{31}$ Department of Community Medicine, Faculty of Health Sciences, University of Tromsø, The Arctic University of Norway, Tromsø, Norway; ${ }^{32}$ Escuela Andaluza de Salud Pública, Instituto de Investigación Biosanitaria, Universidad de Granada, Granada, Spain; ${ }^{33}$ CIBER de Epidemiología y Salud Pública (CIBERESP), Madrid, Spain; ${ }^{34}$ Escuela Andaluza de Salud Pública. Instituto de Investigación Biosanitaria ibs.GRANADA, Universidad de Granada. Granada, Spain; ${ }^{35}$ Navarra Public Health Institute, Pamplona, Spain; ${ }^{36}$ IdiSNA, Navarra Institute for Health Research, Pamplona, Spain; ${ }^{37}$ Public Health Directorate, Asturias, Spain; ${ }^{38}$ Public Health Division of Gipuzkoa, BioDonostia Research Institute, San Sebastian, Spain; ${ }^{39}$ Department of Surgical and Preoperative Sciences, Umeå University, Sweden; ${ }^{40}$ Department of Clinical Sciences in Malmö, Lund University, Malmö, Sweden; ${ }^{41}$ Cancer Epidemiology Unit, Nuffield Department of Clinical Medicine, University of Oxford, Oxford, United Kingdom; ${ }^{42}$ MRC Epidemiology Unit, Institute of Metabolic Science, University of Cambridge, Cambridge, United Kingdom; Endocrinology, Morbid Obesity and Preventive Medicine, Oslo University Hospital, Oslo, Norway; ${ }^{45}$ Unit of Nutrition and Cancer, Catalan Institute of Oncology (ICO-IDIBELL), Barcelona, Spain; ${ }^{46}$ Genetic Epidemiology Group, International Agency for Research on 


\section{7 *Corresponding Author}

68 Pietro Ferrari, $\mathrm{PhD}$

69 Nutritional Methodology and Biostatistics Group

70 International Agency for Research on Cancer, WHO

71 150, cours Albert Thomas

7269372 Lyon CEDEX 08, France

$73 \quad$ Tel. +33472738031

74 E-mail: ferrarip@iarc.fr

75

\section{$76 \quad$ Keywords}

77 Pancreatic cancer; healthy lifestyle index; population attributable fraction; EPIC; prospective 78 study.

79

80 Abbreviations

81 BMI: Body Mass Index

82 CI: Confidence Interval

83 EPIC: European Prospective Investigation into Cancer and Nutrition

84 HR: Hazard Ratio

85 PC: Pancreatic Cancer

86 PAF: Population Attributable Fraction

87 WCRF/AICR: World Cancer Research Fund/American Institute for Cancer Research

88 WHR: Waist-to-Hip ratio 
90 Background. Pancreatic cancer (PC) is a highly fatal cancer with currently limited 91 opportunities for early detection and effective treatment. Modifiable factors may offer 92 pathways for primary prevention. In this study, the association between the healthy lifestyle 93 index (HLI) and PC risk was examined.

94 Methods. Within the European Prospective Investigation into Cancer and Nutrition (EPIC) 95 cohort, 1,113 incident PC (57\% women) were diagnosed from 400,577 cancer-free participants 96 followed-up for 15 years (median). HLI scores combined smoking, alcohol intake, dietary 97 exposure, physical activity and, in turn, overall and central adiposity using BMI (HLI $\mathrm{BMI}_{\text {) }}$ and waist-to-hip ratio (WHR, HLIwHR), respectively. High values of HLI indicate adherence to 99 healthy behaviors. Cox proportional hazard models with age as primary time variable were 100 used to estimate PC hazard ratios (HR) and 95\% confidence intervals (CI). Sensitivity analyses 101 were performed by excluding, in turn, each factor from the HLI score. Population attributable fractions (PAF) were estimated assuming participants' shift to healthier lifestyles.

103 Results. The HRs for a one-standard deviation increment of HLIBMI and HLIWHR were 0.84 $104\left(95 \%\right.$ CI: $\left.0.79,0.89 ; \mathrm{p}_{\text {trend }}=4.3 \mathrm{e}-09\right)$ and $0.77\left(0.72,0.82 ; \mathrm{p}_{\text {trend }}=1.7 \mathrm{e}-15\right)$, respectively. 105 Exclusions of smoking from HLI ${ }_{W H R}$ resulted in HRs of $0.88\left(0.82,0.94 ; p_{\text {trend }}=4.9 \mathrm{e}-04\right)$. The 106 overall PAF estimate was 19\% (95\% CI: 11\%, 26\%), and 14\% (6\%, 21\%) when smoking was 107 removed from the score.

108 Conclusion. Adherence to a healthy lifestyle was inversely associated with PC risk, beyond 109 the beneficial role of smoking avoidance. Public health measures targeting compliance with 110 healthy lifestyles may have an impact on PC incidence. 
112 In the last decades, the rise in pancreatic cancer (PC) incidence has become a major public 113 health concern with mortality rates expected to double by 2030 in American and European 114 populations [1-3]. Commonly diagnosed at late stages, PC is a highly fatal cancer with similar 115 incidence and mortality rates [4]. In the current absence of available screening tools [5], the 116 identification of modifiable risk factors might be important for PC prevention.

117 The World Cancer Research Fund/American Institute for Cancer Research (WCRF/AICR) 118 international expert panel estimated that at least one-third of all cancers could have been 119 prevented through lifestyle management including diet, obesity and physical activity habits [6]. PC incidence rates are nearly four times higher in high-income countries such as the United States and Western European countries than in middle- and low-income countries [4], suggesting that PC occurrence may be associated with lifestyle factors specifically prevalent in the Western world. Individual examination of lifestyle risk factors of PC have led to the identification of smoking, as well as body fatness, adult attained height, type- 2 diabetes, and heavy alcohol drinking as positive risk factors, while diet and physical activity have been inconsistently associated with PC risk $[7,8]$. There is limited evidence regarding the joint association of different lifestyle factors on PC incidence, especially among European populations $[9,10]$.

Previous epidemiological studies have identified clusters of modifiable exposures, assessable through a priori scores reflecting compliance with primary prevention guidelines [11], which were evaluated in relation to cardiovascular diseases $[12,13]$, cancer incidence $[14,15]$, and overall and cause-specific mortality $[16,17]$. A multi-component score termed the Healthy Lifestyle Index (HLI), combining information on smoking, alcohol intake, dietary habits, body mass index (BMI), and physical activity has been previously related to colorectal [18], breast [19], gastric [20], and overall cancers [21] within the European Prospective Investigation into 
136 Cancer and Nutrition (EPIC) study. Within the American Association of Retired Persons

137 (AARP) study a strong inverse association was observed between the HLI and PC risk[9].

138 In this work, the association between the HLI and PC risk was examined within the EPIC study.

139 Two versions of the score were used, i.e. (i) with BMI to reflect overall adiposity and (ii) with

140 waist-to-hip ratio to reflect central adiposity. The marginal role of single factors in the HLI

141 score was investigated, particularly smoking. Population attributable fractions were also

142 estimated.

\section{Material and Methods}

145 Study population. EPIC is a multicenter prospective study designed to investigate the etiology 146 of cancer in relation to diet and other lifestyle factors [22]. From 1992 to 2000, 521,324

147 participants aged from 35 to 70 years were recruited across 10 European countries, mostly from 148 the general population, of which 70\% were women. Exceptions were the French cohort (school 149 and university employees), the Spanish and Italian centers (blood donors), Utrecht and 150 Florence centers (breast cancer screening participants), and Oxford (vegetarians and 'health 151 conscious' participants). In France, Utrecht and Naples women only were recruited. Study 152 participants provided informed consent before completing questionnaires at baseline. 153 Participants from Norway were excluded from this study, as information on physical activity 154 was not compatible with the other centers [23].

155 Cancer cases were identified during follow-up based on population cancer registries in 156 Denmark, Italy, Netherlands, Spain, Sweden, and the United Kingdom, and on a combination 157 of methods, including health insurance records, contacts with cancer and pathology registries, 158 and active follow-up of EPIC participants and their next of kin in France, Germany, and Greece. 
159 Mortality data were collected from, either the cancer or mortality registries at the regional or national level.

161 The most recent vital status and cancer diagnosis update were used. Vital status was known for $98.4 \%$ of all EPIC subjects, while $1.6 \%$ of participants emigrated, withdrew or were lost to follow-up. The current follow-up period ended as follows: December 2009 in Varese and Murcia, December 2010 in Florence, Ragusa, Turin, Asturias, Bilthoven and Utrecht, December 2011 in Granada, Navarra, San Sebastian and Cambridge, December 2012 in Oxford, Umeå, and Denmark, and December 2013 in Malmö. The end of follow-up was considered as the last known contact with participants in France (June 2008), Heidelberg and Potsdam (December 2009), and Naples (December 2010) and Greece (December 2012). Cases of PC were primary incident tumor of the pancreas, coded according to the International Classification of Diseases $\left(10^{\text {th }}\right.$ edition), which included all invasive pancreatic cancers $(\mathrm{C} 25.0-\mathrm{C} 25.3, \mathrm{C} 25.7-\mathrm{C} 25.9)$. Endocrine and neuroendocrine tumors of the pancreas (C25.4) were censored at date of diagnosis $(n=54)$. Microscopically confirmed PC represented $83 \%$ of the cases $(n=928)$ based on histology of the primary tumor or metastases, cytology or autopsy 174 reports.

175 Exposure assessment. Habitual diet, including alcohol intake, over the year preceding recruitment was assessed at baseline by validated center-specific dietary questionnaires $[22,24]$. Data on anthropometry (self-reported in France and the UK Oxford center) $[25,26]$ physical activity, smoking habits, and prevalent chronic conditions were collected at 179 recruitment through lifestyle questionnaires [22].

180 A diet score was built from the combination of six dietary factors reflecting diet quality [21], 181 i.e. cereal fibers, red and processed meat, the ratio of polyunsaturated to saturated fatty acids, 182 margarine (to express industrially produced trans-fats) [27,28], glycemic load, and fruits and 183 vegetables. For each dietary factor, residuals were computed in models with total energy intake 
184 [29], and grouped into country-specific deciles. Individual scores were summed up and categorized into quintiles.

186 The HLI was generated from the combination of five lifestyle factors, namely: diet score, physical activity, smoking status, alcohol consumption and anthropometry. For each factor, scores ranging from 0 to 4 were assigned to increasingly healthier categories, as described in

Figure 1. The HLI was obtained as the sum of scores of each lifestyle factor [19]. As previous evidence on PC etiology identified waist-to-hip ratio, an indicator of central adiposity, as a PC risk factor [30,31], a HLI based on WHR (HLIWHR) was implemented replacing BMI with sexspecific WHR quintiles.

Statistical analysis. From a study population of 521,324 participants, subjects without lifestyle or dietary information $(n=6,902)$, with ratio of estimated energy intake over energy requirement in the top or bottom $1 \%(n=10,241),[32]$ with self-reported prevalent cancer $(\mathrm{n}=24,221)$, with missing follow-up information $(\mathrm{n}=3,800)$, with missing smoking status $(n=15,684)$ or physical activity $(n=65,054)$ were excluded. For analyses with HLIwHR, subjects with missing WHR were also excluded $(n=45,105)$. Country-specific age standardized PC incidence rates (ASR, per 100,000 person-years, PY) were computed using 5-year categories in the range 50 to 70 years and the standard European population.

The association between the HLI and PC incidence was evaluated using multivariable Cox proportional hazard models, with age as the primary time variable, and Breslow's method to handle ties [33]. The time at study entry was age at recruitment, while the exit time was age at cancer diagnosis, death, loss, or end of follow-up, whichever came first. All models were stratified by study center [32], sex and age at recruitment in 1-year categories.

The HLI $_{\mathrm{BMI}}$ and HLI $\mathrm{WHR}_{\mathrm{R}}$ were, in turn, modeled as continuous variables to compute HR estimates for a one-standard deviation (1-SD), corresponding to about three-point increase in the score. Analyses were also carried out in categories (0-4, 5-9, 10-14, 15-20), using the group 

covariates influencing HLI and PC risk [21,34-36], namely education level (no degree/primary

211 school, secondary/technical or professional school, university degree or more, unknown (4\%)),

212 self-reported baseline diabetes status (no, yes, unknown (8\%)), energy intake from non-alcohol

213 sources (continuous), and height (continuous). Additional adjustment for BMI (continuous)

214 was used in models for HLIwHR. HRs were unchanged after women-specific inclusion of menopausal status, ever use of replacement hormonal replacement therapy and number of fullterm pregnancies, thus adjustment for these variables was not pursued. Overall tests for statistical significance of HRs were determined by comparing Wald-test statistics to a $\chi^{2}$ 218 distribution with degree of freedom (dof) equal to the number of categories minus one for 219 evaluation in categories ( $\mathrm{p}_{\text {Wald }}$ ) and dof equal to one as continuous ( $\mathrm{p}_{\text {trend }}$ ). The proportionality of hazards (PH) assumption was evaluated through the Schoenfeld's residuals [37].

Sensitivity analyses were carried out by excluding, in turn, each factor from the HLI scores to identify factors mostly driving the HLI association with PC risk. The excluded component was used as a confounder in the model.

Assuming a causal relationship between HLIWHR and PC risk, population attributable fractions (PAF) were estimated as the reduction in PC incidence that would occur if study participants shifted to the adjacent healthier category of HLI ${ }_{W H R}$, as [38]

$$
P A F=\frac{\sum_{i=1}^{k} R R_{i} c_{i}-\sum_{i=1}^{k} R R_{i} c_{i}^{*}}{\sum_{i=1}^{k} R R_{i} c_{i}}
$$

with $i=1, \ldots, 4$ indexing the HLIwHR categories, $H_{\mathrm{i}}$ and $\mathrm{c}_{\mathrm{i}}$ expressing the hazards ratio and the observed proportion of participants in category $\mathrm{i}$, respectively, and $\mathrm{c}_{\mathrm{i}}{ }^{*}$ the counterfactual proportion of participants, as detailed in Supplementary Table 1. PAF was also computed assuming a counterfactual scenario whereby men adopted women's lifestyle habits. Given the low PC prevalence and under the proportional hazards assumption, HRs were correct 
approximations of risk ratios $\left(\mathrm{RR}_{\mathrm{i}}\right)$. Confidence intervals were obtained using bootstrap sampling [39].

235 The relationship between the HLI and PC risk was estimated by, in turn, sex, European regions 236 (North: Denmark, Sweden; Central: The United Kingdom, The Netherlands, Germany; South: 237 France, Greece, Italy, and Spain), and smoking status (never, former, current). Interactions were evaluated by comparing the difference in log-likelihood of models with and without 239 interaction terms between HLIwHR and, either sex, European region or smoking, to a $\chi^{2}$ 240 distribution, with dof equal to the total number of interaction terms minus one. Although the 241 PH assumption was satisfied, possible selections could operate among study participants within 24215 year of follow-up, and HR estimates can change with age. The pattern of HR for a 1-SD 243 increase of HLIWHR by age was examined using a flexible parametric survival model on the 244 cumulative hazard scale. Restricted cubic splines with 5 internal knots were used to model the 245 baseline hazard using attained age as the time scale and a time-varying coefficient on HLIWHR $246[40]$.

247 To address potential reverse causality, analyses were carried out excluding the first 2 and 5 248 years of follow-up. In analyses excluding smoking from the HLI, HR estimates after adjustment 249 by smoking status (never, former, current), smoking intensity (number of cigarette/day, continuous) and duration of smoking (years, continuous) were examined. Two-sided p-values were used with a 5\% nominal statistical significance. Analyses were performed using Stata 14 [41].

\section{Results}

255 From a total of 400,577 participants (70\% women) followed-up for 15 years (median) and a 256 total of 5,544,627 person-years, 1,113 incident PC cases were diagnosed. Exclusion of subjects 
without information on their WHR led to 1,075 PC cases from a total of 355,472 participants as reported in Table 1. The overall PC ASR was equal to 6.0 per 100,000 person-years, with relatively large and low ASR estimates observed in Germany (9.4 per 100,000 PY) and France (2.1 per 100,000 person-years), respectively. The individual components of the HLI, together with other confounding variables, are described in Table 2. The HLI was inversely related to education, while the prevalence of diabetes at recruitment was stable across HLI categories. The hypothesis of PH assumption was not rejected with p-value equal to 0.24.

264

A 1-SD higher HLI was inversely associated with PC risk, with HR equal to $0.84(95 \% \mathrm{CI}$ : 265 $\left.0.79,0.89, \mathrm{p}_{\text {trend }}=4.3 \mathrm{e}-09\right)$ for $\mathrm{HLI}_{\mathrm{BMI}}$ and $0.77\left(0.72,0.82, \mathrm{p}_{\text {trend }}=1.7 \mathrm{e}-15\right)$ for HLIwHR, as shown in Table 3. These patterns were confirmed for PC HR estimates for analyses in categories, consistently for HLIBMI and HLIWHR.

Results of sensitivity analyses are displayed in Figure 2. After exclusion of smoking status, 269 the HR for a $1-\mathrm{SD}$ increase of $\mathrm{HLI}_{\mathrm{BMI}}$ was $0.94\left(95 \% \mathrm{CI}: 0.88,1.01 ; \mathrm{p}_{\text {trend }}=0.11\right)$, and after exclusions of, in turn, alcohol and BMI, HRs were $0.85\left(0.80,0.91 ; p_{\text {trend }}=6.3 \mathrm{e}-07\right)$ and 0.79 $\left(0.74,0.85 ; p_{\text {trend }}=7.6 \mathrm{e}-12\right)$, respectively. After exclusion of, in turn, smoking, alcohol, waist272 to-hip ratio from the HLIwHR score, HRs were equal to $0.88\left(0.82,0.94 ; p_{\text {trend }}=4.9 \mathrm{e}-04\right), 0.79$ $273\left(0.74,0.84 ; p_{\text {trend }}=7.0 \mathrm{e}-13\right)$ and $0.79\left(0.74,0.85 ; p_{\text {trend }}=3.2 \mathrm{e}-11\right)$, respectively.

PAF estimates for a shift of participants to the adjacent healthier category of HLIwHR was equal to $19 \%$ (95\%CI: 11\%, 26\%) (Table 4). Excluding, in turn, smoking, alcohol and WHR from the HLIwHR showed PAF estimates of $14 \%(6 \%, 21 \%), 19 \%(10 \%, 25 \%)$, and $16 \%(9 \%, 22 \%)$, respectively. PAF were $8 \%(-3 \%, 18 \%)$ for non-smokers at baseline (never and former) and $20 \%(7 \%, 35 \%)$ for current smokers. PAF estimates were $29 \%(16 \%, 37 \%)$ in men, and $13 \%$ $(2 \%, 24 \%)$ in women. Counterfactual scenario whereby men adopted women's lifestyle habits showed a PAF of $13 \%(9 \%, 26 \%)$. 
281 The association between the HLI $\mathrm{WHR}_{\mathrm{H}}$ and PC risk were similar by sex, European region, and smoking status with pheterogeneity equal to $0.35,0.15$ and 0.62, respectively (Figure 3). Although the PH assumption was satisfied, PC HR estimates for HLIWHR showed weaker associations at older ages (Figure 4). Exclusion of the first 2 and 5 years of follow-up did not materially alter HRs. After exclusion of smoking from the HLI and adjustment by smoking status, intensity and duration, HRs were unchanged (not shown).

\section{Discussion}

In this large European prospective study, healthy lifestyle habits expressed as a HLI score were strongly inversely related to the risk of PC. Adherence to healthy behaviors corresponding to a three-point increase in the score was associated with a $16 \%(95 \% \mathrm{CI}: 11 \%, 21 \%)$ lower PC risk for a score that included BMI, and 23\% (18\%, 28\%) lower PC risk for a score based on WHR. These results support the adoption of healthy lifestyles in PC prevention.

294 Scores reflecting dietary and lifestyle habits have become increasingly popular in cancer epidemiology research $[21,42,43]$. In EPIC, scores expressing adherence to either the Mediterranean diet or the WCRF/AICR recommendations have mainly focused on diet, physical activity and anthropometry, and had previously shown null associations with PC risk in both men and women $[44,45]$. Within the NIH-AARP study, a score based on the American Cancer Society recommendations including physical activity, diet, BMI, alcohol, but not smoking, was associated with a 20\% (95\%CI: 3\%, 35\%) lower PC risk in men, comparing the top vs. bottom category, while no association was observed in women [46]. Within the same cohort, an inverse association was observed between HLI and PC, when smoking was added to the score [9].

304 In the current study, a comprehensive evaluation of the association between HLI and PC risk was undertaken using sensitivity analyses. As smoking is an established strong risk factor of 
PC [47], it has been suggested that the association between lifestyle habits and PC might be primarily driven by smoking [45]. In our analysis, HLI was inversely associated with PC risk even after excluding smoking from the score, with a $12 \%$ risk reduction associated with a threepoint (1-SD) increase in the HLI WHR $(95 \% \mathrm{CI}$ : 6\%, 18\%; ptrend $=4.9 \mathrm{e}-04)$. Additionally, in never and former smokers, the PC HR for a three-point increase in the HLI was equal to $0.87(0.79$,

$3110.95 ; p_{\text {trend }}=2.0 \mathrm{e}-03$, data not shown), suggesting the advantage of adopting healthy habits for PC prevention, beyond the benefit of smoking avoidance.

313 Body fatness is also an established risk factor for PC $[8,48]$. A recent pooled analysis concluded that central adiposity during adulthood assessed through waist circumference, or waist-to-hip ratio may also predict PC risk independently from BMI [49]. In our study, HLI based on WHR showed a marginally stronger relationship with PC risk than HLI based on BMI. The subcutaneous truncal adipose tissue has been positively associated with the development of 318 insulin resistance and diabetes [31,50,51], two recognized risk factors for PC [52], and may explain the role of central adiposity, rather than overall adiposity, in PC etiology. Moreover, smoking and alcohol consumption have been previously associated with increasing visceral fat deposition [53,54], which may suggest common pathways between smoking, alcohol consumption and central adiposity in pancreas carcinogenesis.

In our study, the association between HLI and PC was marginally stronger at younger ages compared to older ages. This pattern could be due to a depletion overtime of participants susceptible to PC [55], a phenomenon resulting in an over representation of non-susceptible participants with adverse lifestyle profiles at older ages, thus leading to weaker relationships. Alternatively, HR patterns could be ascribed to study participants' changes towards healthier lifestyle habits related to ageing, or ultimately due to a true causal association indicating that PC benefits could be more substantial if favorable lifestyle habits were adopted at younger ages 
331 This study is to date the first evaluation of the association between a combination of healthy

332 lifestyle factors and PC incidence in European populations, thus corroborating previous

333 evidence from a US study [9]. The strengths of the present study rely on its prospective multi-

334 country design reflecting heterogeneous lifestyle habits. Its large sample size and long follow-

335 up time allowed ascertainment of over a thousand incident PC cases, increasing the statistical power in comparison with the previous EPIC evaluation [44]. Furthermore, associations were unchanged after exclusion of the first years of follow-up. However, this study also has limitations. First, measurement errors likely affected dietary and lifestyle assessments, possibly introducing bias in estimated associations. Furthermore, as EPIC participants represent a healthy proportion of the general population, risk estimates in our study were likely attenuated.

341 In addition, the evidence for a role of life course socio-economic status on cancer-related risk 342 factors was suggested [57], and the use of education in our study as a proxy for socio-economic 343 status might have introduced residual confounding. Last, our study did not consider potential 344 changes in dietary and lifestyle exposures after recruitment, which could be relevant to estimate 345 the association between lifestyle factors and PC risk, as well as to explain HR patterns over 346 age.

347 Assuming that HLI was causally related to PC risk, and that combinations of different lifestyle

348 factors leading to the same value of the HLI had the same effect on PC risk, PAF estimates 349 indicated that $14 \%$ (95\%CI: $6 \%, 21 \%)$ of PC could have been avoided by controlling central 350 adiposity, alcohol consumption, diet and physical activity, and up to $19 \%(11 \%, 26 \%)$ if smoking control was also implemented, indicating the benefit of adopting healthy lifestyle beyond smoking control. In the AARP study, the PAF was $27 \%$ assuming that participants 353 adopted the healthiest lifestyle pattern [9], while in a recent Australian PC study considering 354 only smoking and BMI, the PAF was $30 \%$ [58]. 


\section{Conclusion}

357 In conclusion, our findings provide evidence that adherence to a combination of healthy 358 lifestyle habits was strongly inversely associated with PC risk in European adults. Inverse 359 associations were observed even after dismissing, in turn, smoking, alcohol drinking, and 360 adiposity. Adherence to healthy lifestyle habits, especially from younger ages, could be an

361 effective primary prevention strategy to control the incidence of $\mathrm{PC}$, a fatal cancer with no 362 screening tools currently available for early detection. 


\section{Financial disclosure}

This work was supported by the Direction Générale de la Santé (French Ministry of Health) (Grant GR-IARC-2003-09-12-01), by the European Commission (Directorate General for Health and Consumer Affairs) and the International Agency for Research on Cancer. The national cohorts are supported by the Danish Cancer Society (Denmark); the Ligue Contre le Cancer, the Institut Gustave Roussy, the Mutuelle Générale de l'Education Nationale and the Institut National de la Santé et de la Recherche Médicale (France); the Deutsche Krebshilfe, the Deutsches Krebsforschungszentrum, and the Federal Ministry of Education and Research (Germany); the Hellenic Health Foundation, the Stavros Niarchos Foundation and the Hellenic Ministry of Health and Social Solidarity (Greece); the Italian Association for Research on Cancer and the National Research Council (Italy); the Dutch Ministry of Public Health, Welfare and Sports, the Netherlands Cancer Registry, LK Research Funds, Dutch Prevention Funds, the Dutch Zorg Onderzoek Nederland, the World Cancer Research Fund and Statistics Netherlands (the Netherlands); the Health Research Fund, Regional Governments of Andalucýa, Asturias, Basque Country, Murcia (project 6236) and Navarra, Instituto de Salud Carlos III, Redes de Investigacion Cooperativa (RD06/0020) (Spain); the Swedish Cancer Society, the Swedish Scientific Council and the Regional Government of Skåne (Sweden); Cancer Research UK (14136 to EPIC-Norfolk; C570/A16491 and C8221/A19170 to EPICOxford), Medical Research Council (1000143 to EPIC-Norfolk, MR/M012190/1 to EPICOxford) (United Kingdom), the Stroke Association, the British Heart Foundation, the Department of Health, the Food Standards Agency, and the Wellcome Trust (UK). This work was part of Sabine Naudin's PhD at Claude Bernard Lyon I University (France), funded by Région Auvergne Rhône-Alpes, ADR 2016 (France). 


\section{Conflict of interest}

None to declare.

\section{Acknowledgments}

We thank Carine Biessy and Bertrand Hemon for their technical support and contribution to this work, as well as all the participants of the EPIC cohort.

\section{Copyright statements}

Where authors are identified as personnel of the International Agency for Research on Cancer / World Health Organization, the authors alone are responsible for the views expressed in this article and they do not necessarily represent the decisions, policy or views of the International Agency for Research on Cancer / World Health Organization.

\section{Data sharing statement}

Information to submit an application to have access to EPIC data and/or biospecimens can be found at http://epic.iarc.fr/access/index.ph. 


\section{References}

1. Rahib L, Smith BD, Aizenberg R, Rosenzweig AB, Fleshman JM, Matrisian LM. Projecting cancer incidence and deaths to 2030: the unexpected burden of thyroid, liver, and pancreas cancers in the United States. Cancer Res. 2014;74:2913-21.

2. Malvezzi M, Bertuccio P, Levi F, La Vecchia C, Negri E. European cancer mortality predictions for the year 2014. Ann Oncol Off J Eur Soc Med Oncol ESMO. 2014;25:1650-6.

3. Ferlay J, Partensky C, Bray F. More deaths from pancreatic cancer than breast cancer in the EU by 2017. Acta Oncol Stockh Swed. 2016;55:1158-60.

4. Bray F, Ferlay J, Soerjomataram I, Siegel RL, Torre LA, Jemal A. Global cancer statistics 2018: GLOBOCAN estimates of incidence and mortality worldwide for 36 cancers in 185 countries. CA Cancer J Clin. 2018;68:394-424.

5. Lindquist CM, Miller FH, Hammond NA, Nikolaidis P. Pancreatic cancer screening. Abdom Radiol N Y. 2018;43:264-72.

6. IARC Working Group on the Evaluation of Carcinogenic Risks to Humans. Personal habits and indoor combustions. Volume 100 E. A review of human carcinogens. IARC Monogr Eval Carcinog Risks Hum World Health Organ Int Agency Res Cancer. 2012;100:1-538.

7. Maisonneuve P, Lowenfels AB. Risk factors for pancreatic cancer: a summary review of meta-analytical studies. Int J Epidemiol. 2015;44:186-98.

8. World Cancer Research Fund/American Institute for Cancer Research. Continuous Update Project Expert Report 2018. Diet, nutrition, physical activity and pancreatic cancer. www.dietandcancerreport.org; 2018. 
9. Jiao L, Mitrou PN, Reedy J, Graubard BI, Hollenbeck AR, Schatzkin A, et al. A combined healthy lifestyle score and risk of pancreatic cancer in a large cohort study. Arch Intern Med. 2009;169:764-70.

10. Lucas AL, Bravi F, Boffetta P, Polesel J, Serraino D, La Vecchia C, et al. Adherence to World Cancer Research Fund/American Institute for Cancer Research recommendations and pancreatic cancer risk. Cancer Epidemiol. 2016;40:15-21.

11. Schuit AJ, van Loon AJM, Tijhuis M, Ocké M. Clustering of lifestyle risk factors in a general adult population. Prev Med. 2002;35:219-24.

12. Stampfer MJ, Hu FB, Manson JE, Rimm EB, Willett WC. Primary Prevention of Coronary Heart Disease in Women through Diet and Lifestyle. N Engl J Med. 2000;343:16-22.

13. Kurth T, Moore SC, Gaziano JM, Kase CS, Stampfer MJ, Berger K, et al. Healthy Lifestyle and the Risk of Stroke in Women. Arch Intern Med. 2006;166:1403-9.

14. Catsburg C, Miller AB, Rohan TE. Adherence to cancer prevention guidelines and risk of breast cancer. Int J Cancer. 2014;135:2444-52.

15. Arthur R, Kirsh VA, Kreiger N, Rohan T. A healthy lifestyle index and its association with risk of breast, endometrial, and ovarian cancer among Canadian women. Cancer Causes Control. 2018;29:485-93.

16. Inoue-Choi M, Robien K, Lazovich D. Adherence to the WCRF/AICR guidelines for cancer prevention is associated with lower mortality among older female cancer survivors. Cancer Epidemiol Biomark Prev Publ Am Assoc Cancer Res Cosponsored Am Soc Prev Oncol. 2013;22:792-802. 
17. McCullough ML, Patel AV, Kushi LH, Patel R, Willett WC, Doyle C, et al. Following cancer prevention guidelines reduces risk of cancer, cardiovascular disease, and all-cause mortality. Cancer Epidemiol Biomark Prev Publ Am Assoc Cancer Res Cosponsored Am Soc Prev Oncol. 2011;20:1089-97.

18. Aleksandrova K, Pischon T, Jenab M, Bueno-de-Mesquita HB, Fedirko V, Norat T, et al. Combined impact of healthy lifestyle factors on colorectal cancer: a large European cohort study. BMC Med. 2014;12:168.

19. McKenzie F, Ferrari P, Freisling H, Chajès V, Rinaldi S, de Batlle J, et al. Healthy lifestyle and risk of breast cancer among postmenopausal women in the European Prospective Investigation into Cancer and Nutrition cohort study. Int J Cancer. 2015;136:2640-8.

20. Buckland G, Travier N, Huerta J m., Bueno-de-Mesquita HB, Siersema P d., Skeie G, et al. Healthy lifestyle index and risk of gastric adenocarcinoma in the EPIC cohort study. Int J Cancer. 2015;137:598-606.

21. McKenzie F, Biessy C, Ferrari P, Freisling H, Rinaldi S, Chajès V, et al. Healthy Lifestyle and Risk of Cancer in the European Prospective Investigation Into Cancer and Nutrition Cohort Study. Medicine (Baltimore). 2016;95:e2850.

22. Riboli E, Hunt KJ, Slimani N, Ferrari P, Norat T, Fahey M, et al. European Prospective Investigation into Cancer and Nutrition (EPIC): study populations and data collection. Public Health Nutr. 2002;5:1113-24.

23. Ekelund U, Ward HA, Norat T, Luan J, May AM, Weiderpass E, et al. Physical activity and all-cause mortality across levels of overall and abdominal adiposity in European men and women: the European Prospective Investigation into Cancer and Nutrition Study (EPIC)123456. Am J Clin Nutr. 2015;101:613-21. 
24. Kaaks R, Slimani N, Riboli E. Pilot phase studies on the accuracy of dietary intake measurements in the EPIC project: overall evaluation of results. European Prospective Investigation into Cancer and Nutrition. Int J Epidemiol. 1997;26 Suppl 1:S26-36.

25. Spencer EA, Appleby PN, Davey GK, Key TJ. Validity of self-reported height and weight in 4808 EPIC-Oxford participants. Public Health Nutr. 2002;5:561-5.

26. Spencer EA, Roddam AW, Key TJ. Accuracy of self-reported waist and hip measurements in 4492 EPIC-Oxford participants. Public Health Nutr. 2004;7:723-7.

27. Chajès V, Biessy $\mathrm{C}$, Byrnes $\mathrm{G}$, Deharveng $\mathrm{G}$, Saadatian-Elahi $\mathrm{M}$, Jenab $\mathrm{M}$, et al. Ecological-level associations between highly processed food intakes and plasma phospholipid elaidic acid concentrations: results from a cross-sectional study within the European prospective investigation into cancer and nutrition (EPIC). Nutr Cancer. 2011;63:1235-50.

28. Saadatian-Elahi M, Slimani N, Chajès V, Jenab M, Goudable J, Biessy C, et al. Plasma phospholipid fatty acid profiles and their association with food intakes: results from a crosssectional study within the European Prospective Investigation into Cancer and Nutrition. Am J Clin Nutr. 2009;89:331-46.

29. Willett WC, Howe GR, Kushi LH. Adjustment for total energy intake in epidemiologic studies. Am J Clin Nutr. 1997;65:1220S-1228S; discussion 1229S-1231S.

30. Berrington de González A, Spencer EA, Bueno-de-Mesquita HB, Roddam A, StolzenbergSolomon R, Halkjaer J, et al. Anthropometry, physical activity, and the risk of pancreatic cancer in the European prospective investigation into cancer and nutrition. Cancer Epidemiol Biomark Prev Publ Am Assoc Cancer Res Cosponsored Am Soc Prev Oncol. 2006;15:879-85. 
31. Janssen I, Heymsfield SB, Allison DB, Kotler DP, Ross R. Body mass index and waist circumference independently contribute to the prediction of nonabdominal, abdominal subcutaneous, and visceral fat. Am J Clin Nutr. 2002;75:683-8.

32. Ferrari P, Day NE, Boshuizen HC, Roddam A, Hoffmann K, Thiébaut A, et al. The evaluation of the diet/disease relation in the EPIC study: considerations for the calibration and the disease models. Int J Epidemiol. 2008;37:368-78.

33. Thiébaut ACM, Bénichou J. Choice of time-scale in Cox's model analysis of epidemiologic cohort data: a simulation study. Stat Med. 2004;23:3803-20.

34. Ferrari P, Jenab M, Norat T, Moskal A, Slimani N, Olsen A, et al. Lifetime and baseline alcohol intake and risk of colon and rectal cancers in the European prospective investigation into cancer and nutrition (EPIC). Int J Cancer. 2007;121:2065-72.

35. Ferrari P, Licaj I, Muller DC, Kragh Andersen P, Johansson M, Boeing H, et al. Lifetime alcohol use and overall and cause-specific mortality in the European Prospective Investigation into Cancer and nutrition (EPIC) study. BMJ Open. 2014;4:e005245.

36. Naudin S, Li K, Jaouen T, Assi N, Kyrø C, Tjønneland A, et al. Lifetime and baseline alcohol intakes and risk of pancreatic cancer in the European Prospective Investigation into Cancer and Nutrition study. Int J Cancer. 2018;143:801-12.

37. Schoenfeld D. Partial Residuals for The Proportional Hazards Regression Model. Biometrika. 1982;69:239-41.

38. Barendregt JJ, Veerman JL. Categorical versus continuous risk factors and the calculation of potential impact fractions. J Epidemiol Community Health. 2010;64:209-12. 
39. Efron B, Tibshirani R. Bootstrap Methods for Standard Errors, Confidence Intervals, and Other Measures of Statistical Accuracy. Stat Sci. 1986;1:54-75.

40. Lambert PC, Royston P. Further Development of Flexible Parametric Models for Survival Analysis. Stata J. 2009; Volume 9 Number 2.:21.

41. StataCorp. 2015. Stata Statistical Software: Release 14. College Station, TX: StataCorp LP. 42. Krebs-Smith SM, Pannucci TE, Subar AF, Kirkpatrick SI, Lerman JL, Tooze JA, et al. Update of the Healthy Eating Index: HEI-2015. J Acad Nutr Diet. 2018;118:1591-602.

43. Reedy J, Wirfält E, Flood A, Mitrou PN, Krebs-Smith SM, Kipnis V, et al. Comparing 3 Dietary Pattern Methods-Cluster Analysis, Factor Analysis, and Index Analysis-With Colorectal Cancer RiskThe NIH-AARP Diet and Health Study. Am J Epidemiol. 2010;171:479-87.

44. Romaguera D, Vergnaud A-C, Peeters PH, van Gils CH, Chan DSM, Ferrari P, et al. Is concordance with World Cancer Research Fund/American Institute for Cancer Research guidelines for cancer prevention related to subsequent risk of cancer? Results from the EPIC study. Am J Clin Nutr. 2012;96:150-63.

45. Molina-Montes E, Sánchez M-J, Buckland G, Bueno-de-Mesquita HB, Weiderpass E, Amiano P, et al. Mediterranean diet and risk of pancreatic cancer in the European Prospective Investigation into Cancer and Nutrition cohort. Br J Cancer. 2017;116:811-20.

46. Kabat GC, Matthews CE, Kamensky V, Hollenbeck AR, Rohan TE. Adherence to cancer prevention guidelines and cancer incidence, cancer mortality, and total mortality: a prospective cohort study. Am J Clin Nutr. 2015;101:558-69. 
47. Lynch SM, Vrieling A, Lubin JH, Kraft P, Mendelsohn JB, Hartge P, et al. Cigarette Smoking and Pancreatic Cancer: A Pooled Analysis From the Pancreatic Cancer Cohort Consortium. Am J Epidemiol. 2009;170:403-13.

48. Rawla P, Thandra KC, Sunkara T. Pancreatic cancer and obesity: epidemiology, mechanism, and preventive strategies. Clin J Gastroenterol. 2019;12:285-91.

49. Genkinger JM, Kitahara CM, Bernstein L, Berrington de Gonzalez A, Brotzman M, Elena JW, et al. Central adiposity, obesity during early adulthood, and pancreatic cancer mortality in a pooled analysis of cohort studies. Ann Oncol. 2015;26:2257-66.

50. Patel P, Abate N. Body Fat Distribution and Insulin Resistance. Nutrients. 2013;5:201927.

51. Taylor R. Insulin Resistance and Type 2 Diabetes. Diabetes. 2012;61:778-9.

52. Song S, Wang B, Zhang X, Hao L, Hu X, Li Z, et al. Long-Term Diabetes Mellitus Is Associated with an Increased Risk of Pancreatic Cancer: A Meta-Analysis. PloS One. 2015;10:e134321.

53. Chiolero A, Faeh D, Paccaud F, Cornuz J. Consequences of smoking for body weight, body fat distribution, and insulin resistance. Am J Clin Nutr. 2008;87:801-9.

54. Molenaar EA, Massaro JM, Jacques PF, Pou KM, Ellison RC, Hoffmann U, et al. Association of lifestyle factors with abdominal subcutaneous and visceral adiposity: the Framingham Heart Study., Association of Lifestyle Factors With Abdominal Subcutaneous and Visceral Adiposity: The Framingham Heart Study. Diabetes Care Diabetes Care. 2009;32, $32: 505,505-10$.

55. Hernán MA. The Hazards of Hazard Ratios. Epidemiol Camb Mass. 2010;21:13-5. 
56. White MC, Holman DM, Boehm JE, Peipins LA, Grossman M, Henley SJ. Age and Cancer Risk. Am J Prev Med. 2014;46:S7-15.

57. Akinyemiju T, Ogunsina K, Okwali M, Sakhuja S, Braithwaite D. Lifecourse Socioeconomic Status and Cancer-Related Risk Factors: Analysis of the WHO study on Global Ageing and Adult Health (SAGE). Int J Cancer. 2017;140:777-87.

58. Wilson LF, Antonsson A, Green AC, Jordan SJ, Kendall BJ, Nagle CM, et al. How many cancer cases and deaths are potentially preventable? Estimates for Australia in 2013. Int J Cancer. 2018;142:691-701. 


\section{Figures Captions}

Fig 1 Scoring system implemented to combine the 5 lifestyle factors into the Heathy Lifestyle Index based on the waist-to-hip ratio (HLI $\left.\mathrm{WHR}_{\mathrm{H}}\right)$

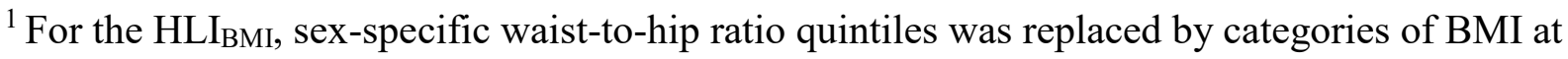
baseline using cut-offs as (4) $22-23.9 \mathrm{~kg} \cdot \mathrm{m}^{-2}$, (3) $24-25.9 \mathrm{~kg} \cdot \mathrm{m}^{-2}$, (2) $<22 \mathrm{~kg} \cdot \mathrm{m}^{-2}$, (1) $26-$ $29.9 \mathrm{~kg} \cdot \mathrm{m}^{-2}$, and $(0)>30 \mathrm{~kg} \cdot \mathrm{m}^{-2}$. 
Fig 2 Hazard ratio estimates for the associations between a 1-SD increment of $\mathrm{HLI}^{1}$ and PC risk after recalculation of the $\mathrm{HLI}_{\mathrm{BMI}}$ and the HLIWHR excluding, in turn, each lifestyle factor

${ }^{1}$ One Standard deviation corresponded to about 3 units of either HLI $\mathrm{BMI}_{\mathrm{BM}}$ or HLI $\mathrm{WHR}_{\text {; }}$

${ }^{2}$ Models evaluating associations between the $\mathrm{HLI}_{\mathrm{BMI}}$ and PC risk were adjusted for education level, diabetes status, non-alcohol energy intakes, height, and the index components currently excluded from the calculation of the HLI, and stratified by study center, age and sex;

${ }^{3}$ Models evaluating associations between the HLIwHR and PC risk were adjusted for education level, diabetes status, non-alcohol energy intakes, height, BMI and the index components currently excluded from the calculation of the HLI, and stratified by study center, age and sex. 
Fig 3 Heterogeneity in the relationship between HLIwHR and PC by sex, European region, and smoking status, expressed for a 1-SD increase of HLIWHR ${ }^{1}$

${ }^{1}$ One Standard deviation corresponded to about 3 units of either $\mathrm{HLI}_{\mathrm{BMI}}$ or HLI $\mathrm{WHR}_{\mathrm{W}}$;

${ }^{2}$ Northern Europe included Denmark and Sweden, Central Europe included United Kingdom, The Netherlands and Germany, and Southern Europe included France, Greece, Italy and Spain; ${ }^{3}$ Models were computed using the HLIwHR excluding smoking;

${ }^{4}$ Models included interaction terms between HLIWHR and, in turn, sex, European region, and smoking status at recruitment. Differences in HRs were assessed comparing the log-likelihood of models with and without interaction terms to a $\chi^{2}$ distribution with degrees of freedom equal to the number of categories minus one. 
Fig 4 Hazard ratio function (and 95\%CI) ${ }^{1}$ for the association between HLIWHR and PC risk over years of age, for 1-SD increase of HLIWHR

${ }^{1}$ Obtained from a flexible parametric survival model using restricted cubic splines with 5 internal knots and a time-varying coefficient on HLIwHr. Model was adjusted for educational level, BMI, height, non-alcohol energy intake, diabetes status, sex, country, age at recruitment. 